\title{
Blood Pressure Variability and Hemorrhagic Transformation after Intravenous Thrombolysis in Acute Ischemic Stroke
}

\author{
Hanna Choi, Seo Young Choi, Jae Guk Kim, Sung-Yeon Sohn, Do-Hyung Kim, Soo Joo Lee* \\ Department of Neurology, Eulii University Hospital, Eulii University School of Medicine, Daejeon, Republic of Korea
}

Article Info

\section{Article Notes}

Received: October 09, 2016

Accepted: November 11, 2016

\section{*Correspondence:}

Dr. Soo Joo Lee, MD, PhD

Department of Neurology, Eulji University Hospital, Eulji University School of Medicine, Dunsan Seo-ro 95, Seo-gu, Daejeon, 35233, Republic of Korea, TelePhone: +82-42-6113430, Fax: +82-42-611-3858; Email: sjoolee@eulji.ac.kr

(c) 2016 Lee SJ. This article is distributed under the terms of the Creative Commons Attribution 4.0 International License

\section{ABSTRACT}

The effectiveness of intravenous tissue plasminogen activator (t-PA) is well established in hyperacute ischemic stroke. Despite its efficacy, spontaneous intracranial hemorrhage after t-PA is a severe complication associated with poor prognosis. We evaluated the role of blood pressure (BP) and BP variability, measured before and after injection of t-PA within 24 hours. Herein, 116 patients were enrolled in this study. BP (systolic blood pressure, diastolic blood pressure, and pulse pressure) were recorded before t-PA and every hour after t-PA for 24 hours. The BP profiles were characterized by initial, mean, maximum (max), minimum ( $\min )$, max-min, and standard deviation (sd). The intracranial hemorrhage was assessed via computed tomography, 24-36 hours after injection of t-PA. The hemorrhagic transformation was classified using clinical and radiological criteria as follows: hemorrhagic transformation $(\mathrm{HT})$, parenchymal hemorrhage $(\mathrm{PH})$, and symptomatic hemorrhage $(\mathrm{SH})$. The intracranial hemorrhage occurred as follows: HT 25.52\% $(n=25), \mathrm{PH} 10.81 \%$ $(n=12), S H 3.60 \%(n=4)$. The PPsd during the 24 -hour period post-injection (24h PPsd) was significantly higher in patients with HT $(14.57 \pm 0.76$ vs. $11.84 \pm 0.39$, 95\% confidence interval $[\mathrm{Cl}] 1.07-4.40, \mathrm{p}<0.001)$ and $\mathrm{PH}(16.74 \pm 4.17$ vs. $11.93 \pm 3.48,95 \% \mathrm{Cl} 2.65-6.97, \mathrm{p}<0.001)$. The odds ratio per $5 \mathrm{mmHg}$ of 24h-PPsd was 2.41 (95\% Cl 1.23-4.72) in $\mathrm{HT}$ and $4.76(95 \% \mathrm{Cl} 1.60-12.17)$ in $\mathrm{PH}$. The variability in pulse pressure during the first 24 hours may be associated with hemorrhagic transformation after thrombolytic therapy with t-PA in hyperacute infarction.

\section{Introduction}

Intravenous injection of tissue plasminogen activator (t-PA) is known to be an effective treatment for acute ischemic stroke patients. In 1995, the study by the National Institute of Neurological Disorder and Stroke [NINDS] t-PA Stroke Study group reported that treatment with t-PA within 3 hours of an ischemic stroke attack improves prognosis ${ }^{1}$. This NINDS study provided the foundation for the t-PA-based treatment protocol. $\mathrm{t}$-PA is the most aggressive treatment among well-known ischemic stroke drugs used to unclog cerebral blood vessels, and recent studies suggesting that the drug might still be effective within 4.5 hours of the initial attack ${ }^{2}$ will likely increase the frequency of t-PA usage.

However, the thrombolytic function of t-PA can induce critical conditions such as cerebral hemorrhage. Results from the NINDS study also showed that $6-7 \%$ of the patients experienced critical hemorrhage. Cerebral hemorrhage is a critical complication that strongly affects the prognosis of the patient, and other studies suggest that up to $10-20 \%$ of the patients could suffer from this 
complication $^{3}$. To minimize the incidence of complications, indications for patients under t-PA are strictly monitored. Based on the NINDS study, cerebral infarction patients who experienced an attack within 3 hours before the treatment were administered $0.9 \mathrm{mg} / \mathrm{kg}$ of t-PA. Moreover, before t-PA injection, multiple conditions need to be checked for, including no sign of hemorrhage on computed tomography (CT), reasonable lesion size, no previous history of stroke or acute myocardial infarction within past few months, and upper limit for the systolic pressure of $185 \mathrm{mmHg}$ and diastolic pressure of $110 \mathrm{mmHg}$.

Despite the proven efficacy of t-PA, the relatively high frequency of potential cerebral hemorrhage is drawing many scientists to perform further research on t-PA usage to reduce the incidence of complications and improve efficacy. Many studies have explored the association between blood pressure maintenance before and after injection and prognosis, since blood pressure is the only risk factor that can be controlled by medication.

During the early stages of cerebral infarction, blood pressure can vary significantly. Blood pressure increases in the early stages, and follows a "U" shaped temporal pattern ${ }^{4}$. Moreover, it varies notably between individuals, and can easily change depending on other factors (such as hospital conditions). Blood pressure is also represented differently, including systolic and diastolic pressures, difference between systolic and diastolic pressures, and changes in blood pressure over time. There are several on-going studies aiming to understand the type of blood pressure that needs to be controlled and the timing of the intervention, to reduce the frequency of cerebral hemorrhage after t-PA treatment and improve prognosis.

Among studies that are examining the relationship between t-PA treatment and prognosis of acute ischemic stroke patients, the European Cooperative Acute Stroke Study (ECASS) is currently being carried out in Europe. This ECASS study, similar to the NINDS study, is a largescale clinical study, currently in Phase 3, to evaluate altering factors such as amount or injection period of t-PA throughout the study period. Yong et al. reported the effect of blood pressure before and after t-PA injection on cerebral hemorrhage and patient prognosis, using the patient cohort in the ECASS study. The results obtained with the cohort from the first ECASS study indicated that Higher systolic BP or diastolic BP at baseline were associated with favorable outcome assessed on modified mRS at 90 days, lower within-patient 0 - to 72-hour average SBP or DBP implied favorable outcome, and reduced variability of 0 - to 72-hour DBP profile was an independent predictor of favorable outcome ${ }^{5}$. The results obtained with the cohort from the second ECASS study showed that higher systolic pressure before t-PA injection, higher maximum/average systolic pressure for 24 hours post-injection, and larger changes in systolic pressure were associated with higher frequency of cerebral hemorrhage and worsening of neurological symptoms by day 7 from the initial attack ${ }^{6}$. However, the first and second ECASS study cohorts had different standards from the NINDS study or the currently suggested t-PA injection standard (i.e. 6 hours within initial attack as standard, injection amount of $1.1 \mathrm{mg} / \mathrm{kg}$ ), and this might result in falsely higher frequency of cerebral hemorrhage.

In this study, we aimed to (1) assess hyperacute ischemic stroke patients within 3 hours of attack (2) measuring and calculate blood pressure before and after intravenous t-PA injection, and (3) identify potential factors that might result in cerebral hemorrhage.

\section{Subjects and Methods}

\section{Subjects and risk factors}

The analysis was in a cohort of 116 ischemic stroke patients admitted to the emergency center of Eulji University Hospital and treated with intravenous t-PA injection, between January 2011 and July 2013. Clinical information of the patients was collected retrospectively using data from stroke patients' database and medical records. Most current international guidelines, based on the ECASS III data recommend the use of rtPA within 4.5 hours. In my country the guideline of the national health insurance changed the time window up to 4.5 hours since January 2014. The study was under old insurance guideline with time window up to 3 hours.

The study protocol has been approved by the institute's committee on human research. All patients were treated with intravenous t-PA injection within 3 hours of initial attack $(0.9 \mathrm{mg} / \mathrm{kg})$, and brain computed tomography (CT) was performed prior to treatment to ensure no prior hemorrhage. Blood pressure was measured every hour after t-PA treatment, for 24 hours. No additional antiplatelet agent or anticoagulant was injected.

Data on patient's age, sex, and previous medical history were collected. Among previous conditions, high blood pressure group was defined as the patients with previously diagnosed high blood pressure or higher than 140/90 mmHg even after the patient was relieved of symptoms. Diabetics were defined as previously diagnosed diabetic patients or had blood sugar level of $126 \mathrm{mg} / \mathrm{dL}$ or above before meals and $200 \mathrm{mg} / \mathrm{dL}$ or above after meals ${ }^{7}$. Atrial fibrillation group was defined as the patients with previous diagnosis or who had evidence of atrial fibrillation on the electrocardiogram while admitted. Cardiac embolic source other than atrial fibrillation group was defined as patients who had reduced cardiac wall motion or cardiac output due to various conditions including heart failure, dilated cardiomyopathy, or coronary artery diseases. Current smokers were defined as the patients who had smoking 
experience in the past 5 years. Initial sugar level was noted, and categorization of stroke was performed using TOAST (trial of Org 10172 in acute stroke treatment) method $^{8}$. The National Institute of Health Stroke Scale (NIHSS) score at the time of admission and t-PA injection time after symptoms occurred were also included as risk factors.

\section{Measurement and analysis of blood pressure}

All blood pressure measurements were performed on the patient in a supine position, with an automated device on the non-paralyzed upper arm. If systolic and diastolic pressures before and after the injection were higher than $185 \mathrm{mmHg}$ and $110 \mathrm{mmHg}$, medication was given according to the guidelines ${ }^{4}$. Blood pressures measured right after admissions were defined as initial systolic, diastolic blood pressure and pulse pressure (SBP, DBP, and PP). Later, SBP, DBP, and PP were measured every hour for 24 hours from the t-PA injection. Pulse pressure was defined as the difference between systolic pressure and diastolic pressure (SBP-DBP). Blood pressure-related factors for first 24 hours were categorized into SBP, DBP, and PP, and mean, standard deviation (SD), maximum value (max), minimum value (min), and difference between the maximum and minimum values (max-min) were calculated. Among these measurements, BP variability was represented as standard deviation and difference between the maximum and minimum values of SBP, DBP, and PP.

\section{Analysis of radiological findings}

All patients were checked for hemorrhagic transformation after t-PA injection by performing CT after 24 - 36 hours from the injection. Hemorrhagic transformation was categorized using radiological standards of ECASS ${ }^{9}$. Hemorrhagic infarction (HI) was defined as small petechia inside the infarction lesion or fused petechia, but no space-occupying effect of the lesion due to hemorrhage. Parenchymal hemorrhage ( $\mathrm{PH})$ was defined as visible blood clot. According to the standards of SITS-MOST (the safe implementation of Thrombolysis in Stroke-Monitoring Study), patients with parenchymal hemorrhage that is larger than $30 \%$ of the lesion and worsened clinical symptoms (NIHSS score increased by 4 or more) are categorized into symptomatic hemorrhage $(\mathrm{SH})^{10}$.

Analysis of imaging results for hemorrhagic transformation was completed independently by a neurologist and a neurology resident. If analysis results were inaccurate, they re-performed the analysis until an agreement was reached. Concordance rate was calculated by kappa analysis. The concordance rate between the two professionals was very high, with $\kappa=0.93(\mathrm{p}<0.001)$ for hemorrhagic transformation analysis and $\kappa=0.96$ $(p<0.001)$ for parenchymal hemorrhage analysis.

\section{Statistical Analysis}

Statistical analysis was performed using SPSS 18.0. For comparison between the groups, categorical independent variables (sex, previous medical history, and smoking history) were assessed using Fisher exact test or Chi-square test. Comparison of continuous variables (age, time, blood pressure, and blood sugar level) was performed using Mann-Whitney U test or Student's t-test. The characteristics of SBP, DBP, PP profiles were included respectively in a bivariate analysis, and the known baseline predictors were included in the model to obtain adjusted estimates of the effects of the characteristics of BP profiles on the respective hemorrhagic outcomes ( $\mathrm{HI}$ and $\mathrm{PH}$ ) using multiple logistic regression. The enrolled known predictors are age, initial NIHSS, diabetes, initial glucose, current smoker, cardiac embolism other than atrial fibrillation, and previous stroke history. Results were presented as ORs with $95 \%$ CIs in 5-mm $\mathrm{Hg}$ increase as unit for each characteristic of BP profiles. All the blood pressure-associated factors have a high degree of multicollinearity, and thus could not be applied to a standalone model at once. Therefore, each of the factors were put into the model independently and assessed by regression analysis. Factors that could affect hemorrhage were corrected through multivariate analysis. $\mathrm{P}<0.05$ was considered statistically significant.

\section{Results}

\section{Risk factors and initial blood pressures}

Out of 116 patients, 111 patients were analyzed, after excluding 3 patients who transferred to a different center after intravenous t-PA treatment, and 2 patients with no blood pressure information since they were not admitted to ICU.

The average age of the 111 analyzed subjects was $64.31 \pm 12.52$ years, and there were more male patients (66.7\%; $n=74)$ than female patients $(33.3 \% ; n=37)$. High blood pressure was observed in $65.8 \%(n=73)$ of the patients, $27.9 \%(n=31)$ had diabetes, $35.1 \%(n=39)$ had atrial fibrillation, $18.9 \%(\mathrm{n}=21)$ had cardiac embolic source other than atrial fibrillation, $21.6 \%(n=24)$ had previous history of stroke, and $37.8 \%(n=42)$ were smokers. Depending on different subtypes of cerebral infarction, $30.6 \%(n=34)$ had large artery atherosclerosis, $11.7 \%$ $(n=13)$ had small vessel occlusion, and $37.8 \%(n=42)$ had cardiogenic embolism. In the remaining cases, $0.9 \%(n=1)$ had other determined etiology, and 17.1\% (n=19) had undetermined etiology, while $1.8 \%(n=2)$ of the patients had averted stroke, where lesion was not visible under MRI after 24 hours of t-PA treatment. Median NIHSS score at the time of admission was 9 , with interquartile range (IQR) of 9.9 - 12.5. Blood sugar level was $145.58 \pm 5.56 \mathrm{mg} / \mathrm{dl}$, and average t-PA injection tie was $2.19 \pm 0.59$ hours. 


\begin{tabular}{|c|c|c|c|c|c|c|}
\hline & \multicolumn{3}{|c|}{ Hemorrhagic Transformation } & \multicolumn{3}{|c|}{ Parenchymal Hemorrhage } \\
\hline & $\begin{array}{c}\text { No } \\
(n=86,77.5 \%)\end{array}$ & $\begin{array}{c}\text { Yes } \\
(n=25,22.5 \%)\end{array}$ & $P$-value & $\begin{array}{c}\text { No } \\
(n=99,89.2 \%)\end{array}$ & $\begin{array}{c}\text { Yes } \\
(n=12,10.8 \%)\end{array}$ & $P$-value \\
\hline Male & $59(68.6 \%)$ & $15(60.0 \%)$ & 0.42 & $65(65.7 \%)$ & 9 (75.0\%) & 0.52 \\
\hline Age, year & $66.5(56.0-73.3)$ & $68.0(55.0-75.0)$ & 0.68 & $67.0(55.0-74.0)$ & $67.0(55.5-73.5)$ & 0.31 \\
\hline Hypertension & $56(65.1 \%)$ & $17(68.0 \%)$ & 0.79 & $65(65.7 \%)$ & $8(66.7 \%)$ & 0.61 \\
\hline Diabetes & $21(24.4 \%)$ & $10(40.0 \%)$ & 0.13 & $25(25.3 \%)$ & $6(50.0 \%)$ & 0.07 \\
\hline Atrial fibrillation & $28(32.6 \%)$ & $11(44.0 \%)$ & 0.29 & $34(34.3 \%)$ & $5(41.7 \%)$ & 0.75 \\
\hline $\begin{array}{l}\text { Cardiac embolic source other } \\
\text { than atrial fibrillation }\end{array}$ & $13(15.1 \%)$ & $8(32 \%)$ & 0.08 & $17(17.2 \%)$ & $4(33.3 \%)$ & 0.24 \\
\hline Previous stroke & $16(18.6 \%)$ & $8(32.0 \%)$ & 0.15 & $18(18.2 \%)$ & $6(50.0 \%)$ & $0.01 *$ \\
\hline Current smoker & $31(36.0 \%)$ & $11(44.0 \%)$ & 0.47 & $34(34.3 \%)$ & $8(66.7 \%)$ & 0.05 \\
\hline Initial glucose, $\mathrm{mg} / \mathrm{dL}$ & $\begin{array}{c}124.0 \\
(108.0-163.0)\end{array}$ & $\begin{array}{c}137.0 \\
(109.5-173.5)\end{array}$ & 0.41 & $\begin{array}{c}129.0 \\
(109.0-161.0)\end{array}$ & $\begin{array}{c}143.0 \\
(99.3-227.5)\end{array}$ & 0.48 \\
\hline Initial NIHSS & $8.0(5.0-18.0)$ & $13.0(8.5-18.0)$ & 0.06 & $9.0(5.0-18.0)$ & $12.0(7.3-17.8)$ & 0.52 \\
\hline $\begin{array}{l}\text { Time to IV t-PA from onset, } \\
\text { hours }\end{array}$ & $2.3(1.8-2.8)$ & $2.3(1.7-2.8)$ & 0.68 & $2.3(1.7-2.8)$ & $2.3(1.8-2.8)$ & 0.44 \\
\hline SBPinitial, mmHg & $156.6 \pm 3.44$ & $151.76 \pm 4.85$ & 0.40 & $156.43 \pm 31.01$ & $154.83 \pm 25.47$ & 0.86 \\
\hline DBPinitial, mmHg & $89.28 \pm 2.03$ & $83.44 \pm 2.64$ & 0.15 & $88.72 \pm 18.04$ & $81.75 \pm 15.60$ & 0.20 \\
\hline PPinitial, $\mathrm{mmHg}$ & $68.29 \pm 2.47$ & $68.32 \pm 4.29$ & 0.10 & $67.71 \pm 22.48$ & $73.08 \pm 22.86$ & 0.44 \\
\hline
\end{tabular}

Data are expressed as mean $\pm S D$, median (interquartile range), or $n(\%)$ as appropriate.

SBPinitial, initial Systolic blood pressure; DBPinitial, initial diastolic blood pressure; PPinitial, initial pulse pressure. ${ }^{*} p<0.05$

Table 1: Comparison of demographic data, baseline clinical findings, and initial blood pressure.

CT performed a day after t-PA injection revealed that $25.52 \%(\mathrm{n}=25)$ of the patients had hemorrhagic transformation, while $11.71 \%(\mathrm{n}=13)$ had $\mathrm{HI}, 10.81 \%$ $(\mathrm{n}=12)$ had $\mathrm{PH}$, and $3.60 \%(\mathrm{n}=4)$ had SH. Comparing the two groups with and without hemorrhagic transformation, initial SBP, and DBP, as well as other risk factors had a similar distribution in both groups (table 1). Comparison of the two groups with and without PH showed a higher frequency of previous stroke in the group of patients with PH $(18.2 \%, n=18$ vs. $50 \%, n=6, p=0.01)$. Initial SBP and DBP, as well as other risk factors, had a similar distribution in both groups (table 1).

\section{Comparison of blood pressure-related factors for 24 hours post t-PA injection (table 2, and 3)}

The group with hemorrhagic transformation had a significantly higher standard deviation (PPsd) and maxmin (PPmin-max) values of pulse pressure compared to the group without hemorrhage. (PPsd during 24-hour period, $24 \mathrm{~h}$-PPsd, $14.57 \pm 0.76$ vs. $11.84 \pm 0.39,95 \%$ Confidence interval (CI) 1.07-4.40, p<0.001; PPmax-min during 24hour period, $24 \mathrm{~h}-\mathrm{PPmax}-\min , 55.68 \pm 2.59$ vs. $48.02 \pm 1.79$, 95\% CI 0.53-14.79, $\mathrm{p}=0.04$ )

In the group with PH, PPsd and PPmin-max values were higher than the group without hemorrhage, $(24 \mathrm{~h}-\mathrm{PPsd}$, $16.74 \pm 4.17$ vs. $11.93 \pm 3.48,95 \%$ CI $2.65-6.97, \mathrm{p}<0.001$; 24h-PPmax-min, $61.08 \pm 14.61$ vs. $48.37 \pm 15.77,95 \%$ CI 3.22-22.20, $\mathrm{p}=0.04$ ) and PPmax was also higher (PPmax during 24-hour period, 95\% CI 0.01-22.00, $\mathrm{p}=0.05$ ).

Among PH group, SH group that had worsened clinical symptoms had higher mean and maximum SBP compared to the group without hemorrhage (SBP mean during 24hour period, 137.52 [125-151.9] vs. 121 [119.31-126.25] $\mathrm{p}=0.03$, SBP max during 24-hour period, 161 [147-179] vs. 138.5 [136.25-149.75], $\mathrm{p}=0.03$ ), and PPsd and PPmaxmin values were also higher (24h-PPsd, 16.68 [14.2-22.72] vs. 12.03 [9.37-14.45], $\mathrm{p}=0.01,24 \mathrm{~h}-\mathrm{PP} \max -\mathrm{min}, 67.5$ [5775.7 ] vs. 47 [37-59], $\mathrm{p}=0.03$ ).

Risk factors including age, NIHSS at admission, blood sugar level at admission, and previous medical history were adjusted to calculate the odds ratio (table 3). Systolic and diastolic blood pressure factors were not shown in the table since they were not significant. With $\mathrm{p}<0.05$ as standard, higher PPsd was associated with increased chance of hemorrhagic transformation (Odds ratio per

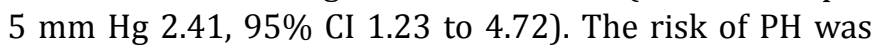
also associated with higher PPsd (Odds ratio per $5 \mathrm{~mm} \mathrm{Hg}$ $4.79,95 \%$ CI 1.60 to 14.17 ), and showed about two-fold increased risk compared to hemorrhagic transformation. With $\mathrm{p}<0.1$ as standard, PPmax-min was also associated with increased risk of PH (Odds ratio per $5 \mathrm{~mm} \mathrm{Hg} \mathrm{1.23,}$ $95 \%$ CI 0.98 to 1.56).

\section{Discussion}

This study aimed to understand the effects of t-PA injection, as well as blood pressure-related factors on hemorrhagic transformation in hyperacute ischemic stroke patients before and after t-PA injection. Prior to t-PA injection, no blood pressure-related factors were associated with hemorrhage. Among blood pressure-related factors after t-PA injection, PPsd and PPmax-min, representing PP 


\begin{tabular}{|c|c|c|c|c|c|c|c|c|c|}
\hline & \multicolumn{3}{|c|}{ Hemorrhagic Transformation } & \multicolumn{3}{|c|}{ Parenchymal Hemorrhage } & \multicolumn{3}{|c|}{ Symptomatic Hemorrhage } \\
\hline & $\begin{array}{c}\text { No } \\
(n=86,77.5 \%)\end{array}$ & $\begin{array}{c}\text { Yes } \\
(n=25,22.5 \%)\end{array}$ & $P$-value & $\begin{array}{c}\text { No } \\
(n=99,89.2 \%)\end{array}$ & $\begin{array}{c}\text { Yes } \\
(n=12,10.8 \%)\end{array}$ & $P$-value & $\begin{array}{c}\text { No } \\
(n=107,96.4 \%)\end{array}$ & $\begin{array}{c}\text { Yes } \\
(n=4,3.6 \%)\end{array}$ & $P$-value \\
\hline \multicolumn{10}{|c|}{ Systolic Blood Pressure } \\
\hline Mean & $138.23 \pm 1.86$ & $136.35 \pm 3.62$ & 0.64 & $138.67 \pm 17.42$ & $130.70 \pm 15.59$ & 0.13 & $121.0(119.3-126.3)$ & $137.5(125.0-151.9)$ & $0.03^{*}$ \\
\hline SD & $12.14 \pm 0.42$ & $11.64 \pm 0.74$ & 0.57 & $11.94 \pm 3.78$ & $12.74 \pm 4.03$ & 0.50 & $8.8(8.2-13.5)$ & $11.4(9.3-14.9)$ & 0.26 \\
\hline Max & $162.21 \pm 2.20$ & $160.88 \pm 3.82$ & 0.29 & $162.49 \pm 20.19$ & $157.08 \pm 19.28$ & 0.38 & $138.5(136.3-149.8)$ & $161.0(147.0-179.0)$ & $0.03 *$ \\
\hline Min & $116.01 \pm 1.71$ & $113.76 \pm 3.26$ & 0.54 & $116.31 \pm 16.06$ & $108.83 \pm 13.33$ & 0.12 & $107.0(90.3-114.0)$ & $114.0(104.0-126.0)$ & 0.19 \\
\hline Max-Min & $46.20 \pm 1.65$ & $47.12 \pm 3.11$ & 0.79 & $46.18 \pm 15.29$ & $48.25 \pm 15.79$ & 0.66 & $33.5(29.3-50.5)$ & $46.0(34.0-57.0)$ & 0.21 \\
\hline \multicolumn{10}{|c|}{ Diastolic Blood Pressure } \\
\hline Mean & $79.24 \pm 1.04$ & $79.62 \pm 2.19$ & 0.87 & $79.29 \pm 9.51$ & $79.67 \pm 13.18$ & 0.90 & $79.3(72.7-85.6)$ & $71.3(65.2-85.7)$ & 0.27 \\
\hline SD & $9.65 \pm 0.38$ & $10.96 \pm 0.57$ & 0.09 & $9.79 \pm 3.47$ & $11.22 \pm 2.96$ & 0.18 & $8.9(7.6-11.8)$ & $9.9(8.3-12.4)$ & 0.58 \\
\hline Max & $98.91 \pm 1.38$ & $101.20 \pm 2.51$ & 0.43 & $99.31 \pm 12.32$ & $100.33 \pm 16.20$ & 0.79 & $100.0(90.0-106.0)$ & $93.0(81.0-107.3)$ & 0.40 \\
\hline Min & $60.88 \pm 1.30$ & $59.56 \pm 2.76$ & 0.64 & $60.87 \pm 12.27$ & $58.52 \pm 14.17$ & 0.49 & $61.0(51.0-69.0)$ & $53.5(41.5-67.0)$ & 0.32 \\
\hline Max-Min & $38.02 \pm 1.68$ & $41.64 \pm 2.68$ & 0.29 & $38.44 \pm 15.42$ & $42.08 \pm 12.27$ & 0.43 & $36.0(28.0-48.0)$ & $40.0(33.5-45.8)$ & 0.56 \\
\hline \multicolumn{10}{|c|}{ Pulse Pressure } \\
\hline Mean & $58.08 \pm 1.63$ & $59.82 \pm 2.54$ & 0.60 & $57.74 \pm 14.98$ & $64.49 \pm 9.26$ & 0.13 & $56.1(47.1-68.4)$ & $67.4(61.2-70.8)$ & 0.14 \\
\hline SD & $11.84 \pm 0.39$ & $14.57 \pm 0.76$ & $<0.001^{*}$ & $11.93 \pm 3.48$ & $16.74 \pm 4.17$ & $<0.001^{*}$ & $12.0(9.4-14.5)$ & $16.7(14.2-22.7)$ & $0.01^{*}$ \\
\hline Max & $82.56 \pm 2.02$ & $87.20 \pm 3.38$ & 0.27 & $82.41 \pm 18.34$ & $93.42 \pm 16.40$ & 0.05 & $83.0(70.0-95.0)$ & $96.5(92.0-104.0)$ & 0.06 \\
\hline Min & $34.53 \pm 1.49$ & $31.52 \pm 2.71$ & 0.34 & $34.04 \pm 14.10$ & $32.33 \pm 10.74$ & 0.69 & $33.0(24.0-42.0)$ & $30.5(24.8-37.0)$ & 0.70 \\
\hline Max-Min & $48.02 \pm 1.79$ & $55.68 \pm 2.59$ & $0.04 *$ & $48.37 \pm 15.77$ & $61.08 \pm 14.61$ & $0.01 *$ & $47.0(37.0-59.0)$ & $67.5(57.0-75.8)$ & $0.03^{*}$ \\
\hline
\end{tabular}

Data are expressed as mean \pm SD

$\mathrm{SD}$, standard deviation; Max, maximum; Min, minimum; Max-Min, maximum - minimum. ${ }^{*} \mathrm{p}<0.05$

Table 2: Comparison of blood pressure profiles derived from measurements obtained during 24 hours after IV t-PA between patients with and those without hemorrhagic transformation, parenchymal hemorrhage, and symptomatic hemorrhage.

\begin{tabular}{|l|c|c|}
\hline & Hemorrhagic Transformation & Parenchymal Hemorrhage \\
\hline Ppmean & $0.95(0.79-1.16)$ & $1.07(0.82-1.40)$ \\
\hline PPsd & $2.41(1.23-4.72)^{* *}$ & $4.76(1.60-14.17)^{* *}$ \\
\hline Ppmax & $1.14(0.98-1.32)$ & $1.16(0.93-1.46)$ \\
\hline Ppmin & $0.87(0.71-1.06)$ & $0.92(0.70-1.21)$ \\
\hline PPmax-min & $0.93(0.82-1.05)$ & $1.23(0.99-1.55)^{*}$ \\
\hline
\end{tabular}

Data are expressed as odds ratio per $5 \mathrm{mmHg}$ (95\% confidential interval) PPsd, standard deviation of PP; PPmax, maximal PP; PPmin, minimal PP; PPmax-min, maximal PP - minimal PP.

Adjusted for age, initial NIHSS, diabetes, initial glucose, current smoker, cardiac embolism other than atrial fibrillation, and previous stroke history. ${ }^{*} p<0.1,{ }^{* *} p<0.05$

Table 3: Effect of pulse pressure (PP) during 24 hours after IV t-PA on hemorrhagic transformation and parenchymal hemorrhage.

variability during a 24-hour period, affected hemorrhagic transformation and PH. The comparison of hemorrhagic transformation group and $\mathrm{PH}$ group revealed a two-fold higher risk, indicating that more severe hemorrhage is associated with higher risk.

The effects of blood pressure-associated factors on the prognosis of stroke patient during the acute phase are currently a topic of interest for many scientists. However, there is no previous study clearly indicating the association between blood pressure-related factors and risk of hemorrhage before thrombolytic treatment. An Australian study reported that the risk of hemorrhage is higher in patients with higher blood pressure pre-treatment when treated with streptokinase ${ }^{11}$. After this study, the majority of t-PA related studies using NINDS as a standard have either excluded such patients from their study or reduced blood pressure with medical intervention if the patient's initial systolic and diastolic pressures were above 185 $\mathrm{mmHg}$ and $110 \mathrm{mmHg}$. Therefore, recent studies on thrombolytic treatments do not include the patients with initial high blood pressure level ${ }^{12}$.

However, even after excluding high blood pressure patients, there is no doubt that blood pressure is one of the important prognostic factors in hyperacute ischemic stroke patients treated with t-PA. Furthermore, recent studies have suggested that systolic pressure before and after t-PA injection affects hemorrhagic transformation and prognosis. As mentioned earlier, the second ECASS study cohort has reported that higher the systolic pressure before and after t-PA injection, higher the risk of $\mathrm{PH}^{7}$. There have also been studies suggesting that risk factor of $\mathrm{PH}$ is not systolic pressure before t-PA injection, but systolic pressure 24 hours post-injection ${ }^{13,14}$.

Unlike previous studies, this study did not identify the association between systolic pressure before and after t-PA injection and PH. However, when SH group was separated from $\mathrm{PH}$ group, the analysis showed that the average and maximum values of systolic pressure 24 hours post-injection were significantly higher compared to the asymptomatic hemorrhagic patient group. Due to the small sample size of SH group, the result might be statistically 
biased. However, these results predict a meaningful association between systolic blood pressure and cerebral hemorrhage when the degree of hemorrhage is higher.

Not only the absolute values of SBP and DBP, but also the BP variability during the 24-hour period post-injection was reported to be associated with larger lesion volume and worsened clinical symptoms ${ }^{14}$. Regardless of t-PA injection, a larger difference in SBP and DBP during the acute phase of ischemic stroke is associated with reduced survival after 3 months ${ }^{15}$, and worsened neurological symptoms within 7 days of admission ${ }^{16}$. Although the measurement method, timing, mathematical calculation of the blood pressure, as well as measures of the patient's prognosis, differed among these studies, the common claim in these papers is that changes in blood pressure are associated with patient's prognosis.

Changes in blood pressure are thought to affect the patient's prognosis due to its association with damaging cerebral autoregulation. Cerebral autoregulation is a physiological response to maintain blood flow within the brain tissue. Unlike other organs, the brain always requires a certain amount of blood flow, and the brain is capable of maintaining a certain amount of cerebral blood flow by controlling cerebrovascular resistance even when the blood pressure of the body changes due to several different reasons. However, the tissue within ischemic stroke lesion has damaged cerebral autoregulation, which allows maintenance of the blood flow. Systemic arterial pressure then exerts a direct effect on cerebral blood flow. Therefore, ischemic penumbra indicates a state hypoperfusion when the blood pressure of the body is reduced or hyperfusion when the blood pressure of the body is increased. The reason behind larger BP variability negatively affecting the patient's prognosis is the changes in blood flow of the ischemic penumbra around the ischemic stroke lesion ${ }^{13,15}$.

The fact that acute phase PP can affect the prognosis after t-PA injection is a novel finding, which had been unknown prior to this study. Framingham's study on the heart showed the tendency of increased SBP and stable or reduced DBP as aging occurs, which leads to increased PP with age. Therefore, PP is thought to be one of the important blood pressure-related factors associated with cardiovascular diseases ${ }^{17}$. Moreover, there have been studies suggesting that higher PP in acute ischemic stroke is associated with poorer long-term prognosis ${ }^{18,19}$.

Even if the absolute values of SBP or DBP are low, a large difference between SBP and DBP can result in pulsatile stretching power to affecting the blood vessels, leading to the increased stiffness of the blood vessels and eventually causing the damage and ruptures ${ }^{19}$. In the study of cerebral hemorrhage after t-PA injection in acute myocardial infarction patients by Selker HP et al. (1994), the authors define this mechanism as the 'Hammer effect'. Under reduced arterial wall compliance, increased PP results in increased pressure on the blood vessels, and damages to the weakened walls of the blood vessels increases the risk of cerebral hemorrhage ${ }^{20}$. Similarly, in this study, increased PP in the tissue that was damaged due to cerebral ischemia might result in the 'Hammer effect', which could eventually damage the walls of the blood vessels. In addition, unwanted side effects of t-PA that was injected for treatment purpose could work in a synergistic manner to cause severe cerebral hemorrhage.

In the same context, tone can predict that not only the absolute value but also the change in PP is a risk factor of cerebral hemorrhage. Larger changes in the PP indicate that regardless of the absolute values of SBP and DBP, the amplitude of change in SBP and DBP in the opposite direction is large. Therefore, damaged tissues from ischemia and ischemic penumbra are not only under the 'Hammer effect' but also under hypo- or hyperfusion state. Therefore, the changes in the PP might be a more important risk factor than the absolute value of the PP.

The limitations of this study are that it is a single-center study, has a small sample size, and is not a randomized trial. Larger-scale studies might further support the findings of this study. Moreover, discrepancies from the timing of cerebral hemorrhage should be considered. The blood pressure can dramatically increase from cerebral hemorrhage regardless of the cause. The exact timing of the cerebral hemorrhage within 24 hours of the initial attack could not be determined in this study. If cerebral hemorrhage occurred right after t-PA injection, it can cause increased SBP or DBP, and eventually overall increase in blood pressure-related factor during a 24-hour period. In the previous studies mentioned above, one of the reasons for increased average and maximum SBP values in the patient group with hemorrhage could be due to this bias. Furthermore, judging the long-term prognosis after t-PA injection through functional examination three months after the initial attack or survival rate analysis one year after the initial attack could be a meaningful future experiment.

In conclusion, the blood pressure-related factors within 24 hours of t-PA treatment may associate hemorrhagic transformation in the patient. Pulse pressure and its changes may be associated with severe hemorrhagic transformation. We believe that the results of this study would contribute to future medical interventions in controlling blood pressure after t-PA injection.

\section{Acknowledgements}

This study was supported by the Bio \& Medical Technology Development Program of the NRF funded by the Korean government, MSIP (No. 2016M3A9B694241). 


\section{References}

1. The National Institute of Neurological Disorder and Stroke rtPA Stroke Study Group. Tissure plasminogen activator for acute ischaemic stroke. N Eng J Med. 1995; 333(24):1581-1587.

2. Hacke W, Kaste M, Bluhmki E, et al. ECASS Investigators Thrombolysis with alteplase 3 to 4.5 hours after acute ischemic stroke. $\mathrm{N}$ Engl J Med. 2008; 359(13):1317-1329.

3. Hacke W, Kaste M, Fieschi C, et al. Intravenous thrombolysis with recombinant tissue plasminogen activator for acute hemispheric stroke. The European Cooperative Acute Stroke Study (ECASS) JAMA. 1995; 274:1017-1025.

4. Leonardi-Bee J, Bath PM, Phillips SJ, et al. IST Collaborative Group Blood pressure and clinical outcomes in the International Stroke Trial. Stroke. 2002; 33:1315-1320.

5. Yong M, Diener HC, Kaste M, et al. Characteristics of blood pressure profiles as predictors of long-term outcome after acute ischemic stroke. Stroke. 2005; 36:2619-2625.

6. Yong M, Kaste M. Association of characteristics of blood pressure profiles and stroke outcomes in the ECASS-II trial Stroke. 2008; 39(2):366-72.

7. Harrison's principle of internal medicine. 17th ed. MacGraw-Hill, 2008.

8. Adams HP Jr, Woolson RF, Clarke WR, et al. Design of the Trial of Org 10172 in Acute Stroke Treatment (TOAST). Control Clin Trials. 1997; 18(4):358-377.

9. Larrue V, Kummer R, Muller A, et al. Risk factors for severe hemorrhagic transformation in ischaemic stroke patients treated with recombinant tissue plasminogen activator. a secondary analysis of the European-Australasian Acute Stroke Study (ECASS II) Stroke. $2001 ; 32: 438-441$

10. Wahlgren N, Ahmed N, Davalos A, et al. SITS-MOST investigators Thrombolysis with alteplase for acute ischaemic stroke in the Safe Implementation of Thrombolysis in Stroke-Monitoring Study (SITSMOST) an observation study. Lancet. 2007; 369:275-282.
11. Gilligan AK, Markus R, Read S, et al. Australian Streptokinase Trial Investigators Baseline blood pressure but not early computed tomography changes predicts major hemorrhage after streptokinase in acute ischemic stroke. Stroke. 2002; 33:2236-2242.

12. Hacke W, Donnan G, Fieschi C, et al. ATLANTIS Trials Investigators ECASS Trials Investigators; NINDS rt-PA Study Group Investigators Association of outcome with early stroke treatment pooled analysis of ATLANTIS ECASS and NINDS rt-PA stroke trials. Lancet. 2004; 363:768-774.

13. Delgado-Mederos R, Ribo M, Rovira A, et al. Prognostic significance of blood pressure variability after thrombolysis in acute stroke. Neurology. 2008; 71(8):552-8.

14. Butcher $\mathrm{K}$, Christensen S, Parsons $\mathrm{M}$, et al. EPITHET Investigators Postthrombolytic blood pressure elevation is associated with hemorrhagic transformation. Stroke. 2010; 41:72-77.

15. Stead L, Gilmore R, Vedula K, et al. Impact of acute blood pressure variability on ischemic stroke outcome. Neurology. 2006; 66:18781881.

16. Sare G, Ali M, Shuaib A, et al. Relationship between hyperacute blood pressure and outcome after ischemic stroke. Data from the VISTA collaboration Stroke. 2009; 40(6):2098-2103.

17. Haider AW, Larson MG, Franklin SS,et al. Systolic blood pressure diastolic blood pressure and pulse pressure as predictors risk for congestive heart failure in the Framingham Heart Study. Ann Intern Med. 2003; 138(1):10-16.

18. Vemmos K, Tsivgoulis G, Spengos K, et al. Pulse Pressure in acute stroke is an independent predictor of long term mortality. Cerebrovasc Dis. 2004; 18:30-36.

19. Tsivgoulis G, Spengos K, Zakopoulos N, et al. Twenty four hour pulse pressure predicts long term recurrence in acute stroke patients. J Neurol Neurosurg Psychiatry. 2005; 76(10):1360-1365.

20. Selker HP, Beshansky JR, Schmid CH, et al. Presenting pulse pressure predicts thrombolytic therapy-related intracranial hemorrhage. Circulation. 1994; 90(4):1657-1661. 